\title{
CoNf- $900466--91$ \\ COHERENT ANTI-STOKES RAMAN SPECTROSCOPIC MEASUREMENT OF AIR ENTRAINMENT IN ARGON PLASMA JETS
}

J. R. Fincke, R. Rodriquez, C. G. Pentecost

Idaho National Engineering Laboratory

EG\&G Idaho, Inc., Idaho Falls, ID

EGG-M- -89489

DE91 001936

\section{ABSTRACT}

The concentration and temperature of air entrained into an argon plasma jet has been measured using coherent ant,i-Stokes Raman spectroscopy (CARS). The flow field is characterized by a short region of well behaved laminar flow near the nozzle exit followed by an abrupt transition to turbulence. Once the transition to turbulence occurs, air is rapidly entrained into the jet core. The location of the transition region is thought to be driven by the rapid cooling of the jet and the resulting increase in Reynolds number.

\section{INTRODUCTION}

In the plasma spray fabrication of coatings the composition of the surroundings into which the plasma jet flows is known to exert considerable influence on the behavior of the jet and can alter the characteristics and quality of the coatings produced [1]. For example, while the plasma spraying of oxides is successfully conducted in oxygen containing environments, tungsten carbide-cobalt, when similarily sprayed thermally decomposes via oxidation of the carbides leading to inferior, carbon deficient coatings $[2,3]$. The presence of oxygen is due to entrainment of the surrounding atmosphere into the plasma flow.

The complexity of the spray process requires a more detailed understanding of chemical reactions at the particle surface, entrainment mechanisms, and the influence of the surrounding atmosphere on both the flow field and the behavior of reactive particles. As an initial step in understanding this process we have begun to investigate the entrainment of the surrounding atmosphere into $\mathrm{plasma}$ jets using coherent anti-Stokes Raman spectroscopy (CARS). This technique is applicable to the measurement of the concentration of any Raman active species, including $\mathrm{O}_{2}, \mathrm{~N}_{2}$, and $\mathrm{CO}$. In the work described here we have chosen $\mathrm{N}_{2}$ as the species to be probed. The CARS spectra of $\mathrm{N}_{2}$ is well known, and the dissociation and ionization characteristics result in non-depleted populations of neutral, molecular nitrogen at temperatures in excess of $6000 \mathrm{~K}$. The initial results on an argon plasma, issuing into a stagnant atmospheric pressure air environment, are presented here. 


\section{MEASUREMENT TECHNIQUE}

Non-intrusive optical diagnostic techniques such as CARS have become standard tools in high temperature flow-field research. CARS has the advantage of high conversion efficiency, a laser-like coherent signal beam for high collection efficiency, excellent fluorescence and luminosity discrimination, and high spatial and temporal resolution. The theory of CARS and its application as a combustion diagnostic tool has been detailed in several reviews [4-6]. A CARS signal, Figure 1, is generated when two laser beams at frequency $w_{1}$, (termed the pump beams) and one laser beam at frequency $w_{2}$ (termed the stokes beam) interact through the third-order nonlinear susceptibility of the medium $x^{(3)}$ to generate an oscillating polarization and thus coherent (laser like) radiation at frequency $w_{3}=2 w_{1}-{ }^{-1} 2$. The intensity of the CARS signal, $I_{3}$, is proportional to:

$$
I_{3} \propto I_{1}^{2} I_{2}(3 x(3))^{2}
$$

where $I_{j}$ is the intensity at frequency $w_{j}$. This relation assumes that the required momentum phase matching is satisfied and that both beams have the same focal diameter. The third-order nonlinear susceptibility,. $x^{(3)}$, which governs CARS can be expressed as:

$$
x^{(3)}=x_{n}+x_{r}
$$

where $x_{n}$ is the nonresonant contribution and $x_{r}$ is the Raman resonant contribution. The nonresonant susceptibility is proportional to the number density of the species present, and is generally a slowly varying
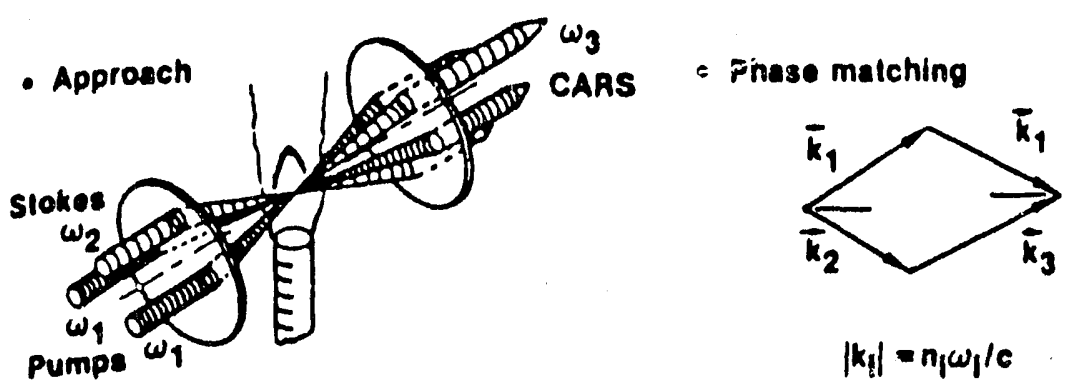

- Enorzy lovel diagrarr.
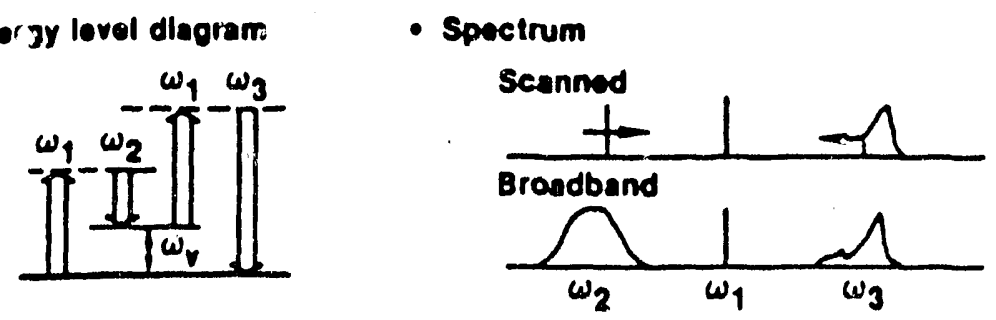

Figure 1. Coherent anti-Stokes Raman spectroscopy. 
function of wavelength. The resonant contribution, far from electronic resonance, is given by:

$$
x^{(3)}=\left(2 c^{4} / h w^{2}\right) N \Sigma\left[\sigma \Delta(v, j) /\left(w_{r}-\left(w_{1}-w_{2}\right)-i G_{v}, j\right)\right]
$$

where $N$ is the number density of the species probed; $\Delta(v, j)$ is the population difference between the states involved in the Raman Transition; $\sigma$ is the Raman scattering cross section; $w_{r}$ is the frequency of a Raman active rotational or vibrational state; and $G_{v, j}$ is the Raman half-width. When the frequency difference $\left(w_{1}-w_{2}\right)$ is close to the frequency of a Raman resonance of a species, $w_{r}$, the magnitude of the CARS radiation at $w_{3}$ is resonantly enhanced, resulting in a signature unique to that molecular species.

CARS spectra can be acquired in two basic ways, figure 1. The scanned approach uses a spectrally-narrow Stokes beam to generate one small segment of the CARS spectrum. The Stokes beam is then scanned across the Raman frequency range to generate a high-resolution CARS spectrum. This method requires considerable time and is limited to temporally stationary flow fields. The broadband approach employs a Stokes source that has a broad spectrum $\left(150-200 \mathrm{~cm}^{-1}\right)$. This allows the entire CARS spectrum to be generated simultaneousij: on the time scale of the laser pulse. This method is preferred for most iractical flow field work, where turbulence and temperature fluctuations are present and temporal fluctuation intensities are of interest. High spatial resolution is achieved by using a crossed beam geometry [6].

The CARS spectrometer consists of a frequency-doubled, Q-switched Nd:YAG 1aser. The second-harmonic green beam is used both as a pump beam for the dye laser which generates the Stokes beam, and as the CARS pump beam. The general beam geometry is shown in Figure 1. The focusing lenses are $100 \mathrm{~mm}$ focal length, resulting in a ellipsoidal measurement volume approximately $200 \mu \mathrm{m}$ in diameter by $1.5 \mathrm{~mm}$ in length. In generating the spatial dependence of concentration and temperature, the plasma torch is moved relative to the stationary CARS measurement volume

Temperatures are derived from the spectral distribution of the CARS signal and concentration is derived from the strength of the signal. In the work performed here, a quick-fit technique [7], based on the full-width at half maximum of the spectral signal, has been used to derive temperatures from spectral data. The temperature determination is calibrated against a platinum, platinum-rhodium thermocouple, while the concentration measurement is calibrated by varying the concentration of air mixed into a cold argon jet. A correction to the measured concentration is derived by assuming vibrational equilibrium and calculating a Boltzmann correction factor using the measured temperature. 
Alternately, spectral fitting procedures such as the CARSFT [8] computer code, or similar technique may be used to derive the same information from data acquired. The uncertainties in the concentration and temperature measurements are estimated to be 6 and 8 percent respectively.

RESULTS

The $1.27 \mathrm{~cm}$. nozzle diameter plasma torch is operated at 20 volts and a current of either 250 or 500 amperes for a total power input of 5 or 10 $\mathrm{kW}$. The torch is 26 and 30 percent efficient at 5 and $10 \mathrm{~kW}$ respectively. The argon gas flow rate is $0.59 \mathrm{SCMH}$, and the atmospheric pressure is $86 \mathrm{kPa}$. Shadowgraphs of the resulting jet at 5 and $10 \mathrm{~kW}$ appear in Figures 2 and 3 . The flow field is characterized by a short region of well behaved laminar flow near the nozzle exit, followed by an abrupt transition to turbulence. The laminar region persists for a slightly longer distance for the higher-power case. The location of the transition region is thought to be driven by the rapid c001ing of the jet and the resulting increase in Reynolds number. The estimated Reynolds number at the torch exit is approximately 100, based on the nozzle diameter. At the location of the transition to turbulence, the Reynolds number is on the order of 10,000. In the higher-power case a hotter and more viscous region maintains the Reynolds number at a lower value for a longer distance, delaying the onset of turbulence.

Contour plots of temperature and relative concentration appear in Figures 4 and 5 for the 5 and $10 \mathrm{~kW}$ cases respectively. The relative concentration is normalized to the number density of $\mathrm{N}_{2}$ at ambient

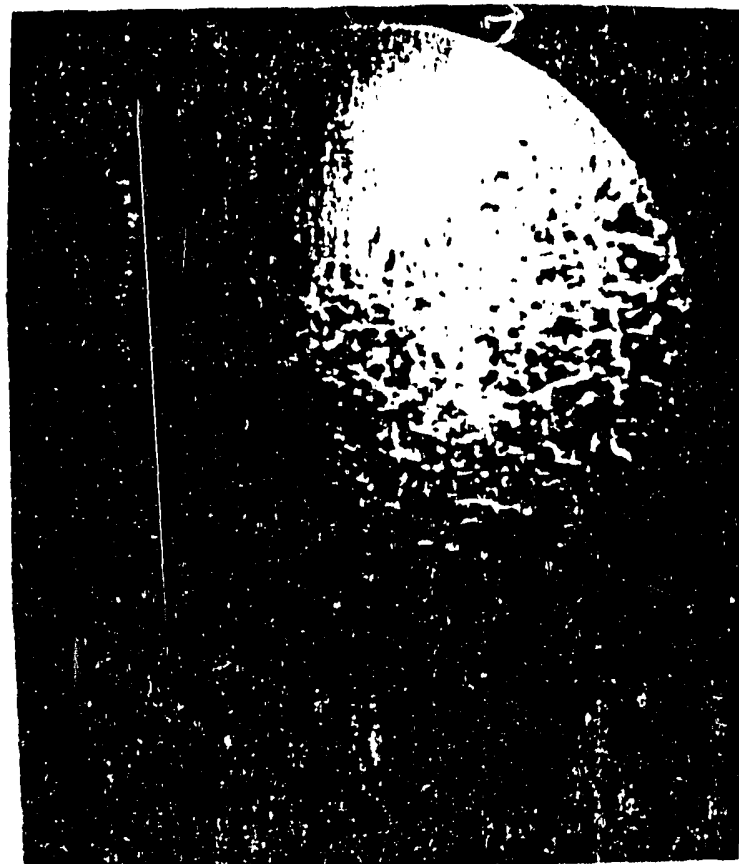

Figure 3. Shadowgraph of plasma jet, $5 \mathrm{~kW}$ torch power.

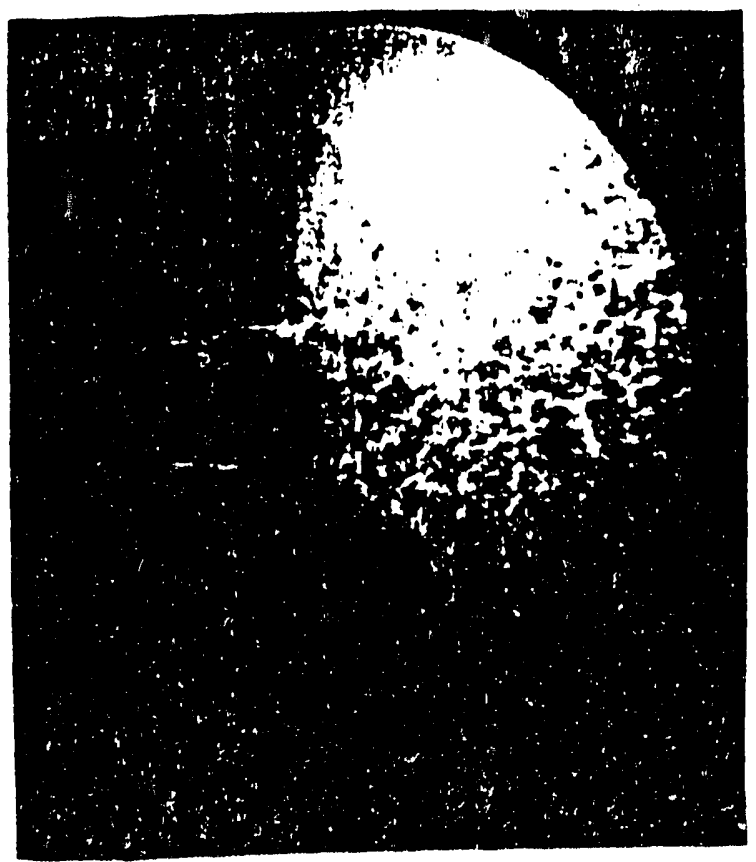

Figure 4. Shadowgraph of plasma jet, $10 \mathrm{~kW}$ torch power. 
conditions; $\mathrm{N}_{0} \approx 1.7 \mathrm{e} 19$. The rapid entrainment of air into the core

flow of the jet, coincident with the onset of turbulence and jet breakup, is evident in the concentration contours. This transition region is not as evident in the associated temperature fields. It is interesting to note that even though the onset of turbulence occurs earlier in the $5 \mathrm{~kW}$ case, a laminar, all-argon core appears to persist for approximately the same distance in both cases and initially the mixing rates are comparable. Further downstream the lower power $5 \mathrm{~kW}$ care spreads more rapidly and entrains air at a greater rate.
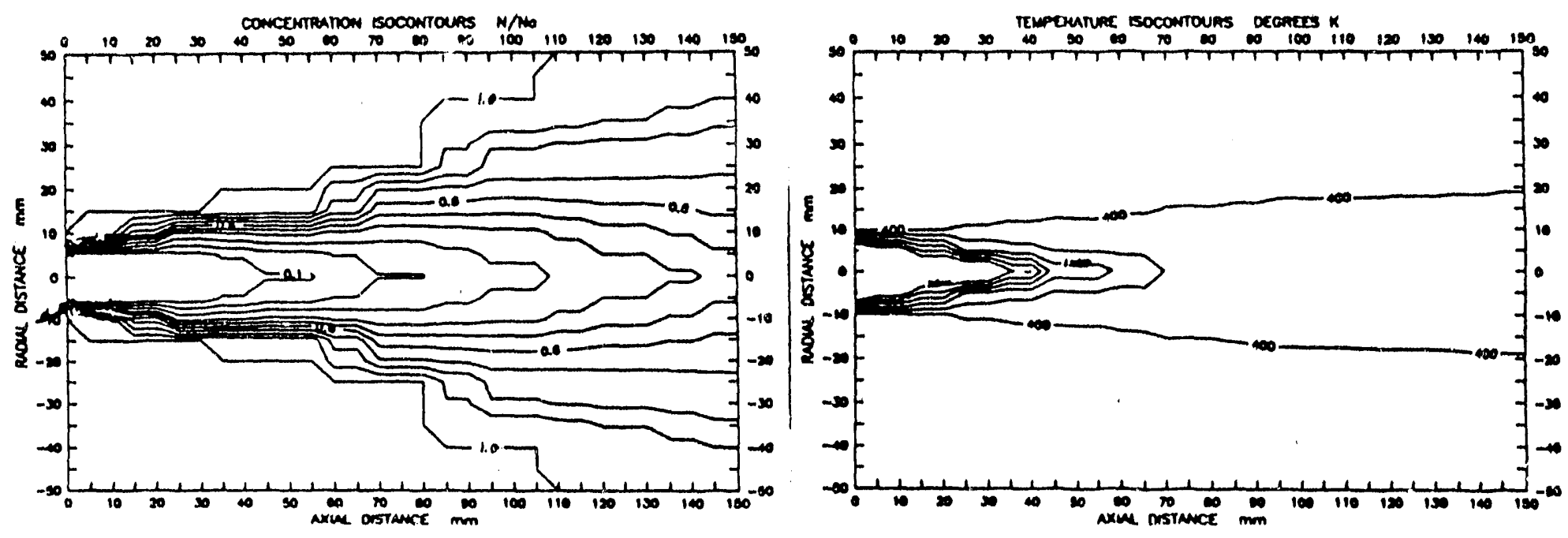

Figure 5. Contour plots of nitrogen concentration and temperature for $5 \mathrm{~kW}$ torch power.
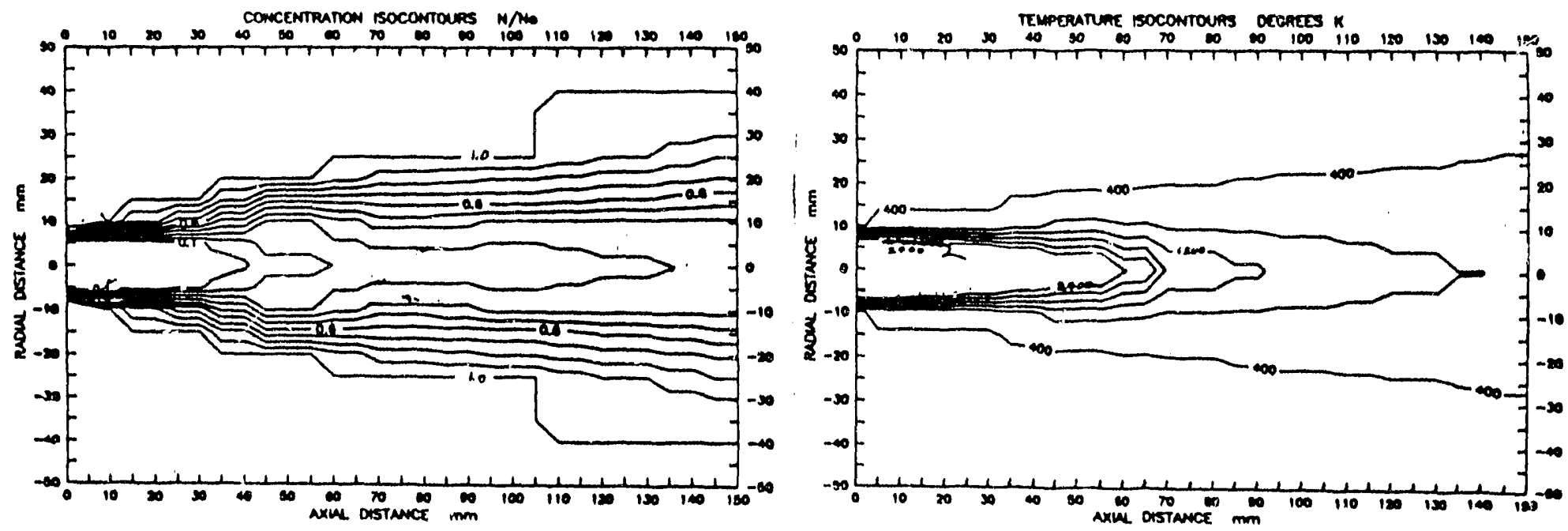

Figure 6. Contour plots of nitrogen concentration and temperature for $10 \mathrm{~kW}$ torch poswer. 


\section{CONCLUSIONS}

The CARS technique has been successfully applied to the measurement of $\mathrm{N}_{2}$ temperature and entrainment in argon plasma jets issuing into stagnant air environments. The entrairment process was found to be characterized by an initial laminar region followed by the rapid onset of turbulence and jet breakup. The location of the transition is dependent on the Reynolds number which is determined primarily by the temperature field. The spreading characteristics of the two cases studied were significantly different, as were ihe measured entrainment rates. The corresponding temperature fields were not as affected by the transition to turbulent flow.

\section{ACKNOWLEDGMENT}

This work was supported by the U. S. Department of Energy, Office of Energy Research, Office of Basic Energy Sciences, U.S. Department of Energy under Contract No. DE-AC07-76ID01570.

\section{REFERENCES}

1. Vardelle, M., Vardelle, A., Roumilhac, Ph., and Fauchais, P., Proceedings of the National Thermal Spray Conference, Cincinnati, $\mathrm{OH}$, pp. 177-121, October 1988.

2. Vinayo, M. E., Kassabji, F., Guyonnet, J., and Fauchais, P., J. Vacuum Science Technology, vol. 6, pp. 2483-2489, 1985.

3. Varacalle, D., J., et al, Symposium Volume of the 1989 TMS/ASM Northeast Regional Meeting, May 1989.

4. Levenson, M. D., Introduction to Nonlinear Laser Spectroscopy, Academic Press, New York, 1982.

5. Antcliff, R. R. and Jarrett, 0 , Review of Scientific Instrumentation, vol. 58, pp. 2075-2080.

6. Eckbreth, A. C. and Anderson, T. J., Applied Optics, vol. 24, pp. $2731-2736,1985$.

7. Eckbreth, A. C., Dobbs, G. M., Stufflebeam, J.H., and Tellex, P. A., Applied Optics, Vol. 23, pp. 1328-1339, 198/.

8. Palmer, R. E, SAND89-8206, Sandia National Laboratories, 1989.

\section{DISCLAIMER}

\footnotetext{
This report was prepared as an account of work sponsored by an agency of the United States Government. Neither the United States Government nor any agency thereof, nor any of their employees, makes any warranty, express or implied, or assumes any legal liability or responsibility for the accuracy, completeness, or usefulness of any information, apparatus, product, or process disclosed, or represents that its use would not infringe privately owned rights. Reference herein to any specific commercial product, process, or service by trade name, trademark, manufacturer, or otherwise does not necessarily constitute or imply its endorsement, recommendation, or favoring by the United States Government or any agency thereof. The views and opinions of authors expressed herein do not necessarily state or reflect those of the United States Government or any agency thereof.
} 
